\title{
Assessment of LAURA for laminar supersonic shallow cavities
}

\author{
William A. Wood, * Maria V. Pulsonetti, ${ }^{\dagger}$ \\ Joel L. Everhart, ${ }^{*}$ and Kim S. Bey \\ NASA Langley Research Center \\ Hampton, VA 23681
}

AIAA Paper 2004-2639

\footnotetext{
* Aerospace Engineer, Aerothermodynamics Branch. Senior AIAA member.

${ }^{\dagger}$ Aerospace Engineer, Aerothermodynamics Branch.

$\ddagger$ Aerospace Engineer, Metals and Thermal Structures Branch.
}

The ability of the LAURA flow solver to predict local heating augmentation factors for shallow cavities is assessed. This assessment is part of a larger effort within the Space Shuttle return-to-flight program to develop technologies to support on-orbit tile repair decisions. The comparison is made against global phosphor thermography images taken in the Langley Aerothermodynamic Laboratory 20-Inch Mach 6 Air Tunnel. The cavities are rectangular in shape, with lengths $L / H$ of $14-20$ and depths $H / \delta$ of 1.1-5.2. The fully laminar results, for $R e_{\theta}=300$, show good agreement between the data sets. For $\operatorname{Re}_{\theta}=503$, the wind tunnel data indicates boundary layer transition with turbulent flow both within and downstream of the cavity. The turbulent flow structures are significantly different from the laminar predictions, with order of magnitude increases in the heating augmentations. Because of the different flow structures, no simple "bump factor" can be used to correct the laminar calculations to account for the turbulent heating levels. A fine gradation in wind tunnel cases will be required to clearly delineate the laminar-to-turbulent transition point, and hence the limits of applicability of the laminar numerical approach.

\section{Nomenclature}

$H \quad$ cavity height, inches

$L \quad$ cavity length, inches

$M_{e} \quad$ Mach number at boundary layer edge

$R e_{\theta} \quad$ Reynolds number based on momentum thickness

$W \quad$ cavity width, inches

$x, y, z \quad$ Cartesian coordinates, inches

$\alpha \quad$ plate inclination, degrees (compression positive)

$\delta \quad$ boundary layer thickness, total enthalpy $99.5 \%$ of free stream, inches

\section{Introduction}

The Space Shuttle return-to-flight program is developing tools to support decisions about repairing thermal protection tile damage while on orbit. Should significant tile damage be observed during future missions, these repair decisions would

This material is declared a work of the U.S. Government and is not subject to copyright protection in the United States. 
need to be made in about a day. Since many technical disciplines are involved, such as aerothermodynamics (including boundary layer transition), thermal protection materials, and thermal structures, the time available for each discipline to analyze the damage situation is measured in hours. Therefore, a heavy reliance will be made upon engineering screening tools to guide the repair decisions.

These engineering screening tools will need to provide functional fits to extensive sets of pre-analyzed damage scenarios. The particular tool to predict heating augmentations inside, and in the near vicinity, of tile damage will be developed primarily based upon global heat transfer measurements obtained using a thermographic phosphor technique ${ }^{1}$ in the Langley Aerothermodynamic Laboratory 20-Inch Mach 6 Air Tunnel. ${ }^{2}$ Although this perfect gas wind tunnel data captures the relevant flow physics phenomena, effects due to the flow chemistry present during the hypersonic orbiter reentry trajectory are not included. Tests in high-enthalpy facilities are underway to determine the relative increments in surface heating due to flow chemistry effects, but the achievable range of flow conditions is more restricted than can be obtained in the perfect gas facility.

Computational fluid dynamic (CFD) simulations are another means to map the perfect gas tunnel results to flight conditions. Further, if the CFD solutions can be obtained fast enough, then CFD can provide a real-time double-check of the engineering tools for a specific damage that may be observed on-orbit. It is recognized that redundancy in terms of personnel, codes, and resources is mandatory should the CFD techniques be needed during a flight emergency, and the project will be more inclusive than just the present authors and the LAURA software used herein. However, any CFD code to be employed in this manner must as a first step be validated for the correct flow physics against the existing damage data sets at the tunnel conditions.

Because of the approximate level of the needed outputs, the strong desire for real-time turn-around, and the granularity present in the experimental data, a true validation ${ }^{3}$ is not being pursued, but rather an assessment for generally reasonable agreement. Also, since the heat conduction through the thermal protection into the structure is a diffusive process, the fine details of the heating augmentations are less important than area-averaged heat rates.

Accordingly, the present investigation assessed the accuracy and range of applicability of the CFD code LAURA to predict local heating augmentations due to shallow cavities representative of missing or damaged orbiter tiles at perfect gas wind tunnel conditions, as a step toward simulating tile damage

\author{
${ }^{1}$ Merski, N. R., "A Relative-Intensity \\ Two-Color Phosphor Thermography \\ System," TM 104123, NASA, 1991

\footnotetext{
${ }^{2}$ Micol, J. R., "Langley Aerothermodynamic Facilities Complex: Enhancements and Testing Capabilities," AIAA Paper 98-0147, Jan. 1998
}

\footnotetext{
${ }^{3}$ Mehta, U. B., "Credible Computational Fluid Dynamics Simulations," AIAA Journal, Vol. 36, No. 5, May 1998, pp. 665-667
} 
scenarios at flight condtions. Because the peak heating during orbiter reentry occurs at laminar flow conditions, laminar calculations were performed. Only rectangular, flow-aligned cavities were considered, matching the currently available tunnel test geometries. Future assessments for cross flow, angled walls, and general gouge cavities will follow the planned wind tunnel tests of these geometries.

\section{Flow Solver}

Numerical simulations were performed using the Langley Aerothermodynamic Upwind Relaxation Algorithm (LAURA). ${ }^{4}$ LAURA is a viscous CFD solver that uses a block-structured domain decomposition with a conservative finite volume formulation. LAURA has been validated against shuttle flight data for external flow aerothermodynamics, ${ }^{5}$ and has been used to assemble wind-side boundary layer maps of the orbiter for reentry trajectories ${ }^{6}$ and for entry cavity studies on a capsule. ${ }^{7}$

The computational grids for the wind tunnel model, described in the next section, had approximately 3 million points, with about $1 / 2$ million points in the cavities; each cavity and flow condition had a distinct grid. The grids external to the cavities used the standard LAURA adaption scheme to align with the bow shock and cluster to the wall, with a target cell Reynolds number of one. Inside the cavities, the spacing at all walls and the shear layer surface matched the wall spacing from the outer grid, with a stretching of 1.2 away from the boundaries. Mesh edge lengths were capped at half an inch.

Solutions were obtained for laminar, full Navier-Stokes, perfect gas air modeling with a constant, uniform wall temperature of $540 \mathrm{R}$.

\section{Wind tunnel data}

The CFD assessment is made against a subset of the wind tunnel data obtained by Everhart ${ }^{8}$ in the Langley Aerothermodynamic Laboratory 20-Inch Mach-6 Air Tunnel on a 28-inch long, 10-inch wide, blunted flat plate with a $1 / 8$-inch nose radius. The tunnel was operated at free stream Reynolds numbers of 2 and 4 million per foot, with the plate at inclinations of zero and 10 degrees compression. The tunnel conditions and corresponding local boundary layer properties are in Table 1. For comparison, on the STS-107 trajectory at free stream Mach numbers above 18, the wind-side boundary layer edge Mach number varies from 2.0-3.5, the momentum thickness Reynolds number varies from 100-700, and the tile thickness to boundary layer thickness ratio varies from $0.5-5.0$. The tile thickness defines the maximum depth of a cavity considered
${ }^{4}$ Gnoffo, P. A., Gupta, R. N., and Shinn, J. L., "Conservation Equations and Physical Models for Hypersonic Air Flows in Thermal and Chemical Nonequilibrium," NASA TP 2867, Feb. 1989

Gnoffo, P. A., "An Upwind-Biased, Point-Implicit Relaxation Algorithm for Viscous, Compressible Perfect-Gas Flows," NASA TP 2953, Feb. 1990

${ }^{5}$ Weilmuenster, K. J. and Gnoffo, P. A., "Solution Strategy for ThreeDimensional Configurations at Hypersonic Speeds," Journal of Spacecraft and Rockets, Vol. 30, No. 4, JulyAugust 1993, pp. 385-394

${ }^{6}$ Gnoffo, P. A. and Weilmuenster, K. J., "Multiblock Analysis for Shuttle Orbiter Re-Entry Heating from Mach 24 to Mach 12," AIAA Paper 93-2813, July 1993

${ }^{7}$ Cheatwood, F. M., Merski, N. R., Riley, C. J., and Mitcheltree, R. A., "Aerothermodynamic Environment Definition for the Genesis Sample Return Capsule," AIAA Paper 2001-2889, June 2001

${ }^{8}$ Everhart, J. L., Bey, K. S., Wood, W. A., and Pulsonetti, M. V., "Experimental and Computational Investigation of Cavity Flow Simulating Debris Damage to Thermal Protection System Tiles," Oral presentation, JANNAF Conference, Dec. 2003

Table 1: Relation between wind tunnel operating conditions and cavity-local boundary layer edge properties.

\begin{tabular}{ccccc}
$R e\left(10^{6} f t^{-1}\right)$ & $\alpha, \operatorname{deg}$ & $M_{e}$ & $R e_{\theta}$ & $\delta$, in \\
\hline 2 & 0 & 2.9 & 300 & 0.14 \\
4 & 10 & 2.25 & 503 & 0.065
\end{tabular}


within the present scope, with deeper penetrations termed as "breaches" and being covered by another team. A literature survey of existing wind tunnel data relevant to the present investigation is included in Reference 8 .

The physical dimensions of the test article cavities that were also simulated with CFD are in Table 2. The cavity widths are included within the table for completeness, though width variations were not investigated. The leading edge of all cavities was 10.5 inches from the virtual sharp nose. Figure 1 shows a representation of the computational test article.

The experimental data is referenced in non-dimensional scaling parameters, so that a given test configuration can map to various locations on the orbiter at different times in the trajectory, depending upon the local boundary layer properties and the specific damage size. The measured data is presented as heating augmentation factors, where a factor of unity equates to the smooth plate heating rate.

\section{$5 \quad$ Results}

Seven cases have been simulated. Solutions for each cavity in Table 2 were run at the 300 and $503 R e_{\theta}$ conditions, except for the length, or $L / H, 20.5$ cavity, which was only run at the $R e_{\theta}=300$ condition.

Historically (see Reference 8), cavities have been classified as either short or long based upon the dominant flow pattern. The outer flow tends to skip over a short cavity, Figure 2, while a long cavity behaves more like separate backward and forward facing steps, Figure 3. The cavities of length 20 are of the long variety. The two shorter $L / H$ cavities are of a length that is intermediate between the depicted canonical cases.

Baseline cavity: $L / H=15.6, R e_{\theta}=300$

For the length 15.6 cavity at $R e_{\theta}=300$ conditions, the cavity depth $H / \delta$ is 1.1. A centerline slice of the heating augmentations from the numerical solution is compared with the experimental measurements in Figure 4, extracted from global surface heat transfer images. Evident is the peak heating on the downstream lip, the lower heating on the cavity floor, and a return to the undisturbed laminar heating rate downstream of the cavity. The extracted measurement data is less smooth than the computed prediction, making specific quantitative comparisons difficult. The overall agreement is good, with the exception of the cavity floor. The phosphor thermography technique calculates heating rates as a function of time-varying temperatures, so in regions of low heating, and hence small temperature variation, the signal-to-noise ratio is low. Such a situation occurs in the cavity floor; the actual floor
Table 2: Cavity physical dimensions.

\begin{tabular}{cccl}
$H$, in & $L / H$ & $W / H$ & variation \\
\hline 0.145 & 15.6 & 2.6 & baseline \\
0.344 & 14.3 & 2.4 & depth \\
0.155 & 20.8 & 2.5 & length \\
0.343 & 20.5 & 2.4 & length and depth
\end{tabular}

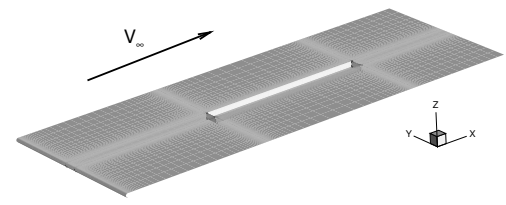

Figure 1: Perspective view of test article numerical model.

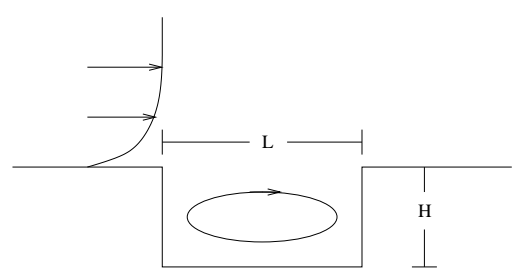

Figure 2: Short, traditionally termed 'open', cavity.

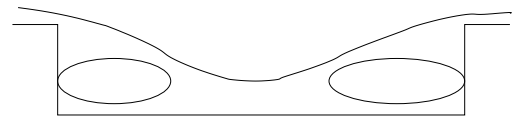

Figure 3: Long, traditionally termed 'closed', cavity.

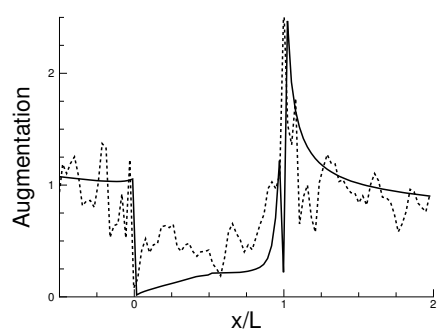

Figure 4: Centerline heating augmentations, $L / H=15.6, \operatorname{Re}_{\theta}=300$. Solid line is computed, dashed line is measured. 
heating can be expected to be lower than the noisy measured data, which the computed heating rates are, but just how much lower cannot be determined.

Because the variation present in the extracted line cuts precludes accurate quantitative comparisons, the remainder of the results are presented as global surface heating images. All of the images share the same contour legend, Figure 5. The darkest blue represents heating levels of one-half the smooth plate value. The other contour levels are spaced at unit increments above the baseline heating level.

The computed and measured global heating augmentation images are shown in Figure 6 as a view from directly above the cavity. The flow is from left to right. The regularly spaced cool dots in the measured image are caused by fiducial marks on the test article. Fiducial marks are present on all the tunnel models, recognizable as regularly spaced irregularities in the images. The fiducial marks are flush with the model surface, and are utilized to map the measured data to a CAD surface during the data reduction. The floor of the cavity has generally $50 \%$ lower heating, rising toward the right-hand end. Downstream of the cavity is a small region of doubled heating rates. The computed result is in good agreement with the measured data for this case.

Depth and length variations at $R e_{\theta}=300$

Comparisons of longer and deeper cavities at the $R e_{\theta}=300$ conditions are shown in Figures 7-10. The deeper cavity, of length 14.3 , has a depth $H / \delta$ of 2.4 , a little more than twice the scaled depth of the prior case. The heating augmentation levels, Figure 7, are very similar to the results of Figure 6, and again the computed and measured data are in good agreement. There is a thin line of higher heating on the downstream lip evident in the measured data. In general, though, the measured data right at the lip has higher uncertainties associated with it, due to several factors: spatial resolution limits; breakdown of the one-dimensional conduction assumption; and errors in the optical view angle correction and mapping to CAD model.
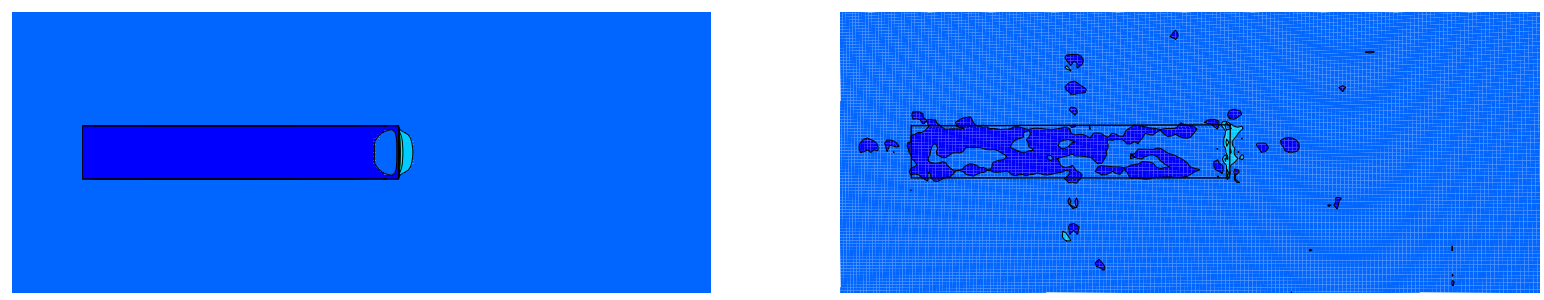

Figure 6: Heating augmentations for baseline cavity, $L / H=15.6, H / \delta=1.1, R e_{\theta}=300$. Left is computed, right is measured. 

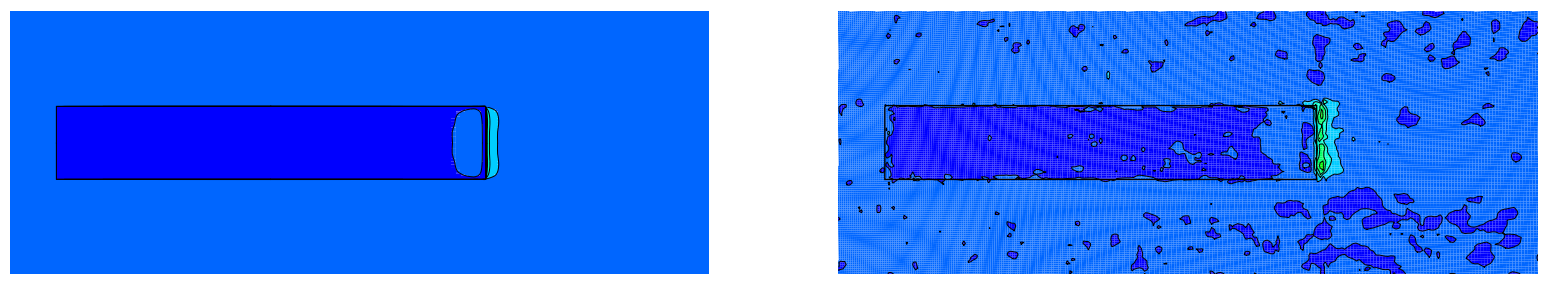

Figure 7: Heating augmentations on deeper cavity, $L / H=14.3, H / \delta=2.4, R e_{\theta}=300$. Left is computed, right is measured.

Grid convergence was checked for this case by running a coarser grid with 200,000 grid points in the cavity, about $60 \%$ of the original grid size. The surface heating augmentations on the coarse grid are shown in Figure 8, which are in agreement with the primary solutions.

The longer, $L / H 20.8$ cavity, Figure 9 , with $H / \delta$ of 1.1 , shows less of a reduction in the cavity floor heating. There is now an increased heating spot at the downstream end of the cavity floor, indicative of the cavity flow field more resembling separate backward and forward facing steps. Trailing downstream from the cavity are heating augmentation streaks of twice the undisturbed value, whose shape suggest laminar vortices from the cavity corners. The two data sets have overall good agreement, while the computed solution predicts higher augmentations right at the trailing edge of the cavity. The resolution of the present experimental data image is not sufficient to allow a detailed comparison of the back face of the cavity, which is not visible at all in the top-down views shown here.

The $L / H 20.5$ cavity, with a depth $H / \delta$ of 2.4 , Figure 10 , is both longer and deeper. The flow field is becoming more complex, with vortices diving down into the cavity, as evidenced by the heating augmentation streaks along the inside edges of the cavity, and then spilling out at the downstream quarter, producing large lobes of elevated heating. The vortices in the computed solution dip in and exit the cavity earlier

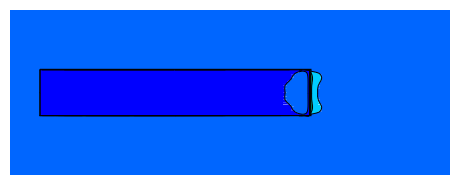

Figure 8: Coarse grid heating augmentations, $L / H=14.3, H / \delta=2.4$, $R e_{\theta}=300$.
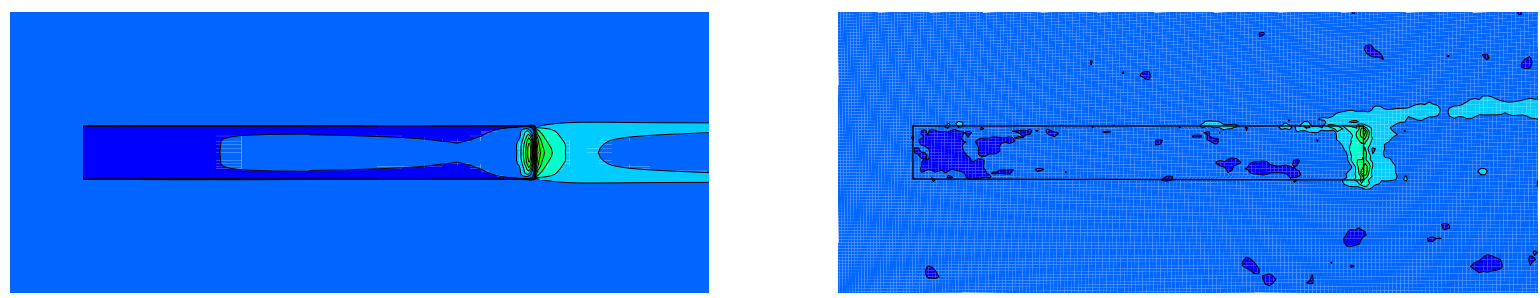

Figure 9: Heating augmentations on longer cavity, $L / H=20.8, H / \delta=1.1, \operatorname{Re}_{\theta}=300$. Left is computed, right is measured. 

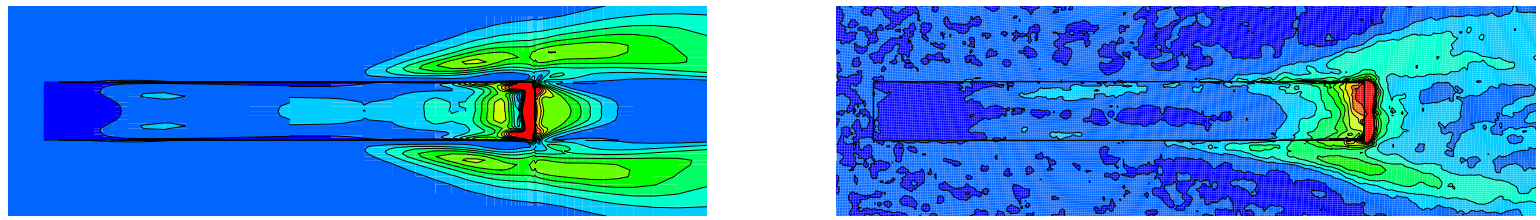

Figure 10: Heating augmentations on deeper and longer cavity, $L / H=20.5, H / \delta=2.4, R e_{\theta}=300$. Left is computed, right is measured.
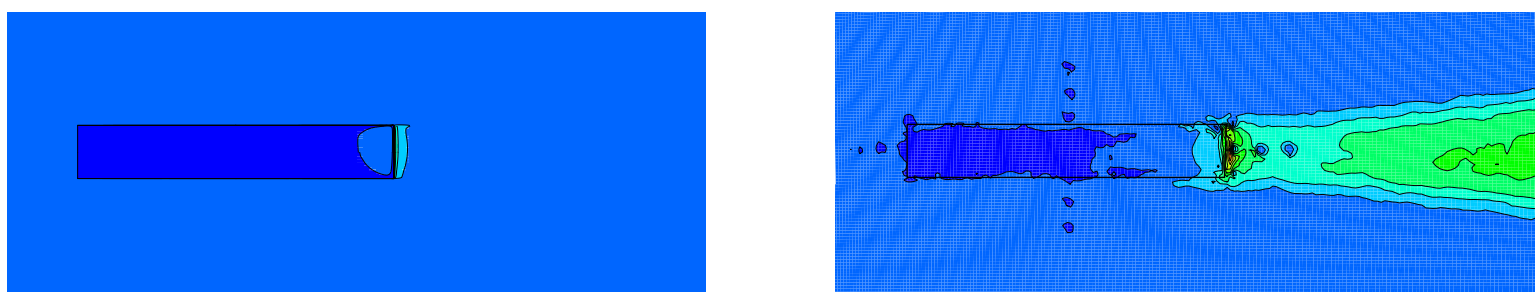

Figure 11: Higher Reynolds number heating augmentations, $L / H=15.6, H / \delta=2.4, R_{\theta}=503$. Left is computed, right is measured.

than is measured, with stronger extra-cavity heating. The computed solution also predicts higher floor heating from the impinging shear layer. The global agreement, though, may still be suitable for the engineering purpose for which it is intended.

Higher Reynolds number, $R e_{\theta}=503$, conditions

The length 15.6 cavity is simulated again, now for the $R e_{\theta}=503$ conditions, Figure 11. The depth is $H / \delta=2.4$, about twice the depth as for the $R e_{\theta}=300$ conditions, due to the thinner boundary layer at the higher Reynolds number. The computed solution shows little difference due to Reynolds number. However, there is a significant difference in the measured heating augmentation downstream from the cavity, with evidence of boundary layer transition and a turbulent wedge. The laminar assumption in the computed solution appears to be sufficient for the internal cavity flow at this condition, but is clearly insufficient for the downstream extracavity flow field. This case has an incipient break down of the laminar assumption, and is an important dividing line in the data set for determining the limits of applicability of the laminar numerical modeling.

The deeper cavity, length 14.3 with $H / \delta=5.2$, shows a dramatic difference between the measured and computed results, Figure 12. Although the boundary layer state is not being directly determined, the measured flow field appears 

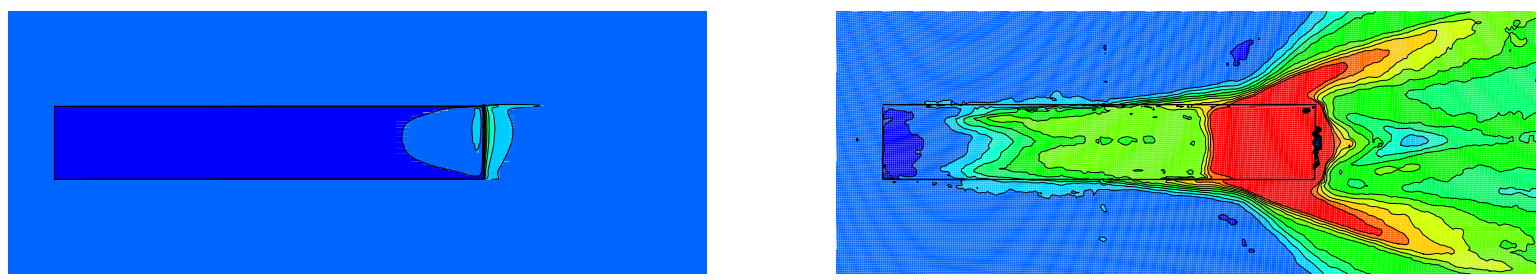

Figure 12: Deeper cavity high Reynolds number heating augmentations, $L / H=14.3, H / \delta=5.2, \operatorname{Re}_{\theta}=503$. Left is computed, right is measured.
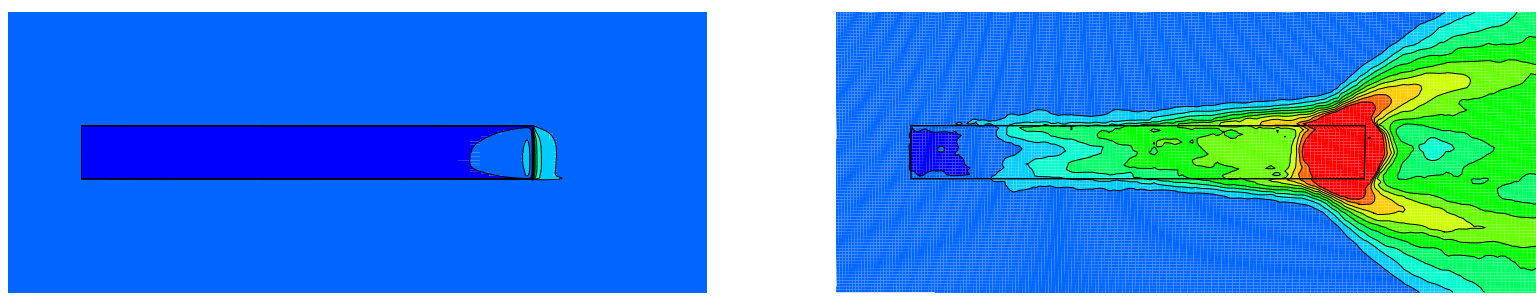

Figure 13: Longer cavity high Reynolds number heating augmentations, $L / H=20.3, H / \delta=2.4, \operatorname{Re}_{\theta}=503$. Left is computed, right is measured.

to be turbulent within and downstream of the cavity, with significantly different flow physics, rendering the laminar computed results irrelevant. The heating augmentations are increased by an order of magnitude both within and without the cavity. As expected, the numerical solution does not give any indication of the break down of the laminar assumption, either in unusual heating patterns or a failure to converge.

Similarly, the longer $L / H 20.8$ cavity, with $H / \delta=2.4$, shows evidence of turbulence in the form of enhanced mixing of the hotter outer flow onto the floor of the cavity, Figure 13. The turbulent flow physics are very different from the laminar prediction, and cannot be approximated by a simple "bump factor" applied to the laminar results.

\section{$6 \quad$ Limitations}

This assessment study is for the perfect gas flow physics of shallow, rectangular cavities in supersonic boundary layers. The effects of reacting chemistry and surface catalysis, which are present at reentry flight conditions, are not modeled. No span-wise pressure gradient or cross flow was included. The cavities were scaled to make the boundary layers in flight and in the tunnel proportional in the similarity parameters, but the actual boundary layer profiles are not necessarily the same between the orbiter in flight and the blunted plate in the tunnel test. The CFD tool was run for steady state assumption and 
does not have a credible boundary layer transition prediction capability.

\section{Conclusions}

The LAURA solver predicts global surface heating augmentations in good agreement with the experimental data set for fully laminar shallow cavities tested in the Langley Aerothermodynamic Laboratory 20-Inch Mach 6 Air Tunnel. Combined with prior validation at high-enthalpies, the code can be used to extrapolate the perfect gas tunnel data to flight conditions, or simulate laminar cavities directly at flight trajectory points. The cavities considered were rectangular in shape and aligned with the flow. The fully laminar cavities ranged in length from $L / H=14-20$, with depths of $H / \delta=1.1$ and 2.4, and were at a local boundary layer condition of $R e_{\theta}=300$.

The laminar solutions performed at $R e_{\theta}=503$ conditions with depths of $H / \delta=2.4$ and 5.2 did not agree with the wind tunnel data, where the wind tunnel images indicate turbulent transition. The turbulent flow structures are significantly different from the laminar structures, and consequently the heating augmentation patterns are very different between the computed and measured results. The numerical solutions at these conditions did not provide any warning of the break down of the laminar assumption, such as a failure to converge, and thus the laminar-to-turbulent limit of applicability for the numerical scheme must be derived from the wind tunnel data set. Since the abrupt transition to turbulence of the free shear layer and recirculation zone inside the cavity causes tremendously increased heating augmentations, a fine gradation of experimental cases is required to clearly delineate the laminar-to-turbulent conditions. An assessment of the turbulent predictive capabilities of CFD codes could be performed against the existing high Reynolds number experimental data set. 